\title{
Effects of probiotic-containing products on stool frequency and intestinal transit in constipated adults: systematic review and meta-analysis of randomized controlled trials
}

\author{
Larry E. Millera, Arthur C. Ouwehand ${ }^{b}$, Alvin Ibarrab \\ Miller Scientific Consulting, Inc., Asheville, NC, USA; DuPont Nutrition and Health, Kantvik, Finland
}

\section{Abstract}

${ }^{a}$ Miller Scientific Consulting, Inc., Asheville, NC, United States (Larry E. Miller); ${ }^{b}$ DuPont Nutrition and Health, Kantvik, Finland (Arthur

C. Ouwehand, Alvin Ibarra)

Conflict of Interest: This work was supported by DuPont Nutrition and Health, Danisco Sweeteners Oy (Kantvik, Finland)

Correspondence to: Larry E. Miller, PhD, Miller Scientific Consulting, Inc., 1854 Hendersonville Road, \#231, Asheville, NC, United States,

e-mail: larry@millerscientific.com

Received 21 March 2017; accepted 24 August 2017; published online 21 September 2017

DOI: https://doi.org/10.20524/aog.2017.0192 and sources of heterogeneity. interpreted with caution.

\begin{abstract}
Background Probiotics are commonly recommended for the alleviation of constipation symptoms. The aim of this research was to determine the effects of probiotic-containing products factors that influence the efficacy of these products.
\end{abstract}

Methods We conducted a systematic review of randomized controlled trials that measured weekly stool frequency or ITT in constipated adults receiving probiotic-containing supplements. A random effects meta-analysis was performed; stool frequency was summarized by the mean erence statistic and ITT was summarized by the standardized mean difference (SMD) statistic. Meta-regression and diagnostic model performance testing were used to identify publication bias

Results A total of 21 studies (23 comparisons) comprising 2656 subjects were included. All studies utilized probiotics containing Lactobacillus or Bifidobacterium species. Probiotic-containing products resulted in a mean increase in weekly stool frequency of 0.83 (95\% confidence interval [CI] $0.53-1.14, \mathrm{P}<0.001)$. There was high heterogeneity among the studies $\left(I^{2}=85 \%, \mathrm{P}<0.001\right)$ and evidence of significant publication bias (Egger's P-value $<0.01$ ). After adjustment for publication ias, the mean difference in weekly stool frequency was reduced from 0.83 to 0.30 . The effects on frequency were greater in studies where functional constipation was diagnosed using Rome III $(\mathrm{P}<0.01)$, or Rome II or III criteria $(\mathrm{P}<0.05)$, compared to non-Rome diagnosis techniques. Probiotic-containing products were also efficacious in reducing ITT ( $\mathrm{SMD}=0.65,95 \% \mathrm{CI} 0.33$ $0.97, \mathrm{P}<0.001)$. There was high heterogeneity among studies $\left(I^{2}=66 \%, \mathrm{P}<0.01\right)$, but no evidence publication bias (Egger's $\mathrm{P}$-value $=0.52$ ). A larger total sample size was associated with greater daily probiotic dosage had no influence on the outcomes.

Conclusion Supplementation with products containing Lactobacillus or Bifidobacterium species increases stool frequency and reduces ITT in constipated adults. However, since significant heterogeneity in outcomes was detected among the studies analyzed, the results should be

Keywords Constipation, meta-analysis, probiotic, stool frequency, transit time

Ann Gastroenterol 2017; 30 (6): 1-11

\section{Introduction}

Functional constipation is a multifactorial syndrome affecting $14 \%$ of adults globally [1]. It is characterized by disordered motility and/or defecation in the absence of any obvious organic etiology. Alterations in the gut microbiota appear to have a role in the development of functional constipation [2,3]. The concentration of Bifidobacterium and Lactobacillus is lower in adults with constipation [4] and the composition of the gut microbiota is associated with gastrointestinal motility [5]. The alterations in gut microbiota 
have not been definitely shown to be a cause or a consequence of constipation. These findings have nonetheless favored the development of hypotheses suggesting that quantitative changes in the gut microbiota are one mechanism by which oral probiotic consumption may contribute to improved symptoms of constipation.

Probiotics are commonly recommended to alleviate gastrointestinal complaints such as constipation, diarrhea, and irritable bowel syndrome. While dozens of clinical studies have been conducted in recent years [6,7], the clinical effects of probiotics are not well characterized. One potential contributing factor is the variability in the diagnostic criteria of gastrointestinal conditions. Although the Rome III criteria were developed over 10 years ago, they remain inconsistently utilized in clinical practice and in clinical trials [8]. In particular, constipation is diagnosed using various criteria, including self-report, Rome II criteria, Rome III criteria, or custom composite definitions that commonly include infrequent bowel movements. Inconsistency in the diagnostic criteria for constipation is but one variable that may introduce variability in the outcomes of probiotic clinical trials. A wide variety of probiotic-containing products are available, with major differences in probiotic strain, dose, and additional ingredients such as synbiotics. While meta-analyses have investigated the efficacy of probiotics in adults with constipation $[9,10]$, none of these studies have thoroughly explored the influence of potentially confounding variables on probiotic efficacy. The purpose of this systematic review and meta-analysis of randomized controlled trials was to determine the efficacy of probiotic-containing products on stool frequency and intestinal transit time (ITT) in constipated adults. A secondary aim of this research was to determine the factors that may influence the efficacy of these products in this population.

\section{Materials and methods}

\section{Literature search}

This study was performed according to the Preferred Reporting Items for Systematic Reviews and Meta-analyses (PRISMA) criteria [11]. We searched MEDLINE and EMBASE for randomized controlled trials of probioticcontaining products in constipated adults that reported stool frequency or ITT, using a combination of diagnostic-specific (bowel movement, colonic, constipation, gastrointestinal, gut, motility, stool, transit) and therapy-specific (probiotic ${ }^{\star}$, symbiotic, lactobacill ${ }^{\star}$, bifidobacteri ${ }^{\star}$, yogurt[yoghurt], fermented milk) keywords. Additionally, manual searches were conducted using the Directory of Open Access Journals, Google Scholar, and the reference lists of included papers and other relevant meta-analyses. No date restrictions were applied to the searches. The final search was conducted on February 10, 2017. The main inclusion criteria were as follows: randomized controlled trial of probiotic-containing supplements; primary diagnosis of functional constipation (by self-report, physician opinion, or symptom-based diagnostic criteria) and/or mean stool frequency less than 3 times per week in the absence of organic disease; non-institutionalized adults with no obvious secondary cause of symptoms (e.g., surgery, chronic disease, opioid medication); and extractable measures of stool frequency and/or ITT over a minimum 7-day supplementation period. Study selection discrepancies between the researchers were resolved by discussion and consensus.

\section{Study selection}

Two researchers independently selected studies for inclusion in the systematic review. Disagreements were resolved by discussion and consensus. Titles and abstracts were reviewed to exclude manuscripts published in non-English journals, review articles, commentaries, letters, and case reports. Fulltext copies of the remaining manuscripts were then retrieved and reviewed. Publications that failed to report weekly stool frequency or ITT, or that described non-randomized, noncontrolled, or otherwise irrelevant studies were also excluded.

\section{Data extraction}

Data were extracted from eligible peer-reviewed articles by one author and then reviewed by a second author. Discrepancies between the researchers were resolved by discussion and consensus. The following variables were recorded in a predesigned database: general manuscript information, study design characteristics, subject characteristics, study quality assessment, and weekly stool frequency and ITT summary statistics required for meta-analysis. Data from the final followup period in each study were utilized in the meta-analysis.

\section{Risk of bias}

The risk of bias for each study was scored according to the presence of three key methodological features: randomization, blinding and subject accountability [12]. Randomization was scored from 0-2, blinding was scored from 0-2, and subject accountability was scored 0 or 1 . Randomized controlled trials with a score of 3-5 were classified as high-quality; studies with a score of 0-2 were classified as low-quality.

\section{Statistical analysis}

We performed a random effects meta-analysis given the apriori assumption of significant heterogeneity in effect sizes due to differences in experimental designs, treatment regimens, and subject characteristics among studies. The statistics of interest were the unstandardized mean difference in stool frequency and the standardized mean difference (SMD) in ITT. Stool frequency was defined as the number of self-reported bowel movements per week. The SMD is a statistic that summarizes outcomes on a common scale and was selected since ITT was 
reported using different units of measure among studies. SMD values of $0.2,0.5$, and 0.8 are defined as small, medium, and large effect sizes, respectively [13]. For each outcome, a positive value implies greater efficacy with probiotic-containing products and a negative value implies greater efficacy with controls. In studies where multiple probiotic groups were compared to a single control group, the sample size of the control group entered into the meta-analysis was adjusted accordingly [14]. Forest plots were developed to illustrate the SMD of individual study findings and the random effects meta-analysis results. Heterogeneity of effects among studies was estimated using the $I^{2}$ statistic, where values of $\leq 25 \%, 50 \%$, and $\geq 75 \%$ represent low, moderate, and high inconsistency, respectively [15]. A one-study-removed meta-analysis was performed to assess the influence of individual studies on the meta-analysis findings. Publication bias was assessed visually with a funnel plot and quantitatively using Egger's test [16]. For each main outcome, random effects meta-regression using the methods of Knapp and Hartung [17] was performed to investigate whether pre-specified covariates explained any of the heterogeneity of treatment effects among studies. Variables selected for model inclusion a priori were age, percentage of female subjects, body mass index, constipation diagnosis method, study location, study sample size, treatment duration, probiotic species, number of probiotic strains, and probiotic dosage. For each covariate in the meta-regression model, the intercept represents the estimated outcome value when the covariate value equals 0 and the slope represents the magnitude of change in the estimated outcome value per unit increase in covariate value. Diagnostic testing of meta-regression findings was performed to assess model performance. Jackknifed residuals were calculated for each study to represent the difference in the meta-regression effect size with and without each study included. A jackknifed residual value $<-3$ or $>3$ for any study was considered an outlier, such that inclusion of the study could significantly alter the main results. All analyses were performed using Comprehensive Meta-analysis software (version 3.3, Biostat, Englewood NJ).

\section{Results}

\section{Study selection}

Our initial database search retrieved 394 titles and abstracts; manual searching relevant bibliographies identified 5 additional records. After screening based on the inclusion criteria, 79 full text articles were reviewed for eligibility. Ultimately, 21 randomized controlled trials [18-38] with 23 comparisons representing 2656 unique subjects were included in the meta-analysis. A flow chart illustrating the study identification and selection is shown in Fig. 1.

\section{Study and subject characteristics}

Study entry criteria were highly variable among studies. Rome III functional constipation was used for diagnosis in 7 studies; the remaining studies used Rome II functional constipation guidelines, modified Rome III functional constipation guidelines, or various combinations of selfreported constipation or infrequent bowel movements, with or without additional criteria based on baseline ITT. Sample sizes ranged from 22-905 subjects for each comparison. Nineteen studies contributed one comparison each and two studies contributed two comparisons: the studies of Eskesen et al [22] and Waller et al [37] assessed two

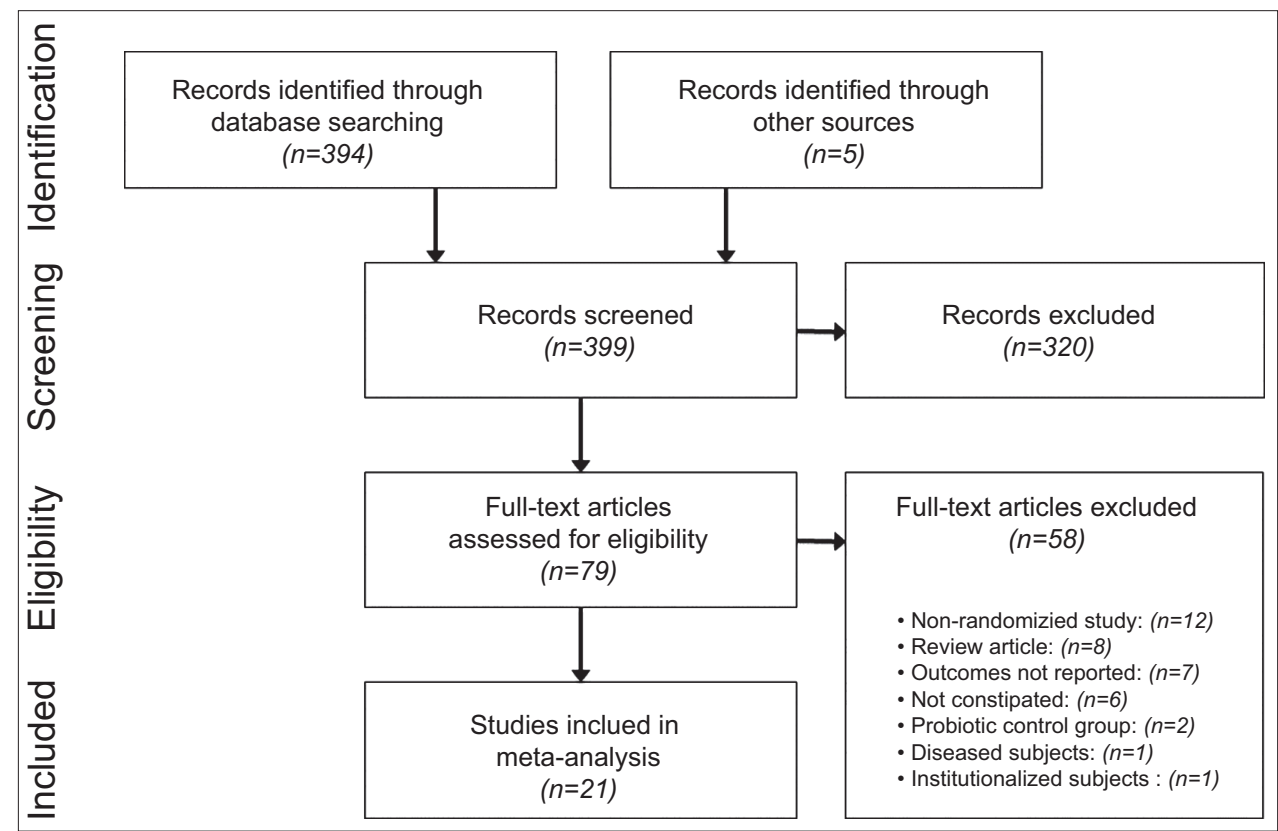

Figure 1 PRISMA study flow diagram 
different dosages of the same probiotic strain. Considerable variability in probiotic daily dosage and treatment duration was observed, and 9 studies included various synbiotics or other dietary ingredients. All studies utilized Lactobacillus or Bifidobacterium, alone or in combination with other probiotic species. Daily probiotic dosages varied considerably among studies, ranging from $0.1 \times 10^{9}$ to $30 \times 10^{9}$ colony forming units (cfu) per day (median $6.5 \times 10^{9} \mathrm{cfu}$ per day). Treatment durations ranged from 7-84 days (median 28 days). Subjects were predominantly female, mean age ranged from 22-50 years, and mean body mass index ranged from $19-32 \mathrm{~kg} / \mathrm{m}^{2}$ (Table 1). A total of 19 studies [19-36,38] with 20 comparisons contributed data for stool frequency and 8 studies [18,21,25,26,30-32,37] with 9 comparisons contributed data for ITT. Intestinal transit time was reported as total intestinal transit time using radiopaque markers in 3 studies, colonic transit time using radiopaque markers in 3 studies, whole gut transit time using radiopaque markers in 2 studies, and orocecal transit time using carmine red dye in 1 study.

\section{Study quality assessment}

Overall, the quality of study reporting was medium-tohigh, with a median total score of 4 (range: 1-5). Nineteen of 23 treatment effects were based on high-quality trials. The method of randomization was inadequately described in most studies. Descriptions of blinding and subject accountability were sufficiently detailed in most studies (Table 2).

\section{Probiotic effects on stool frequency}

Probiotic-containing supplements resulted in a statistically significant improvement in stool frequency versus controls in 11 of 20 comparisons. When pooling data in the meta-analysis, the mean difference in stool frequency per week was 0.83 (95\% confidence interval [CI] 0.53-1.14, $\mathrm{P}<0.001$ ) (Fig. 2). The analogous SMD was 0.49 (95\%CI $0.32-0.66, \mathrm{P}<0.001)$. There was high heterogeneity among studies $\left(I^{2}=85 \%, \mathrm{P}<0.001\right)$ and evidence of significant publication bias (Egger's P-value

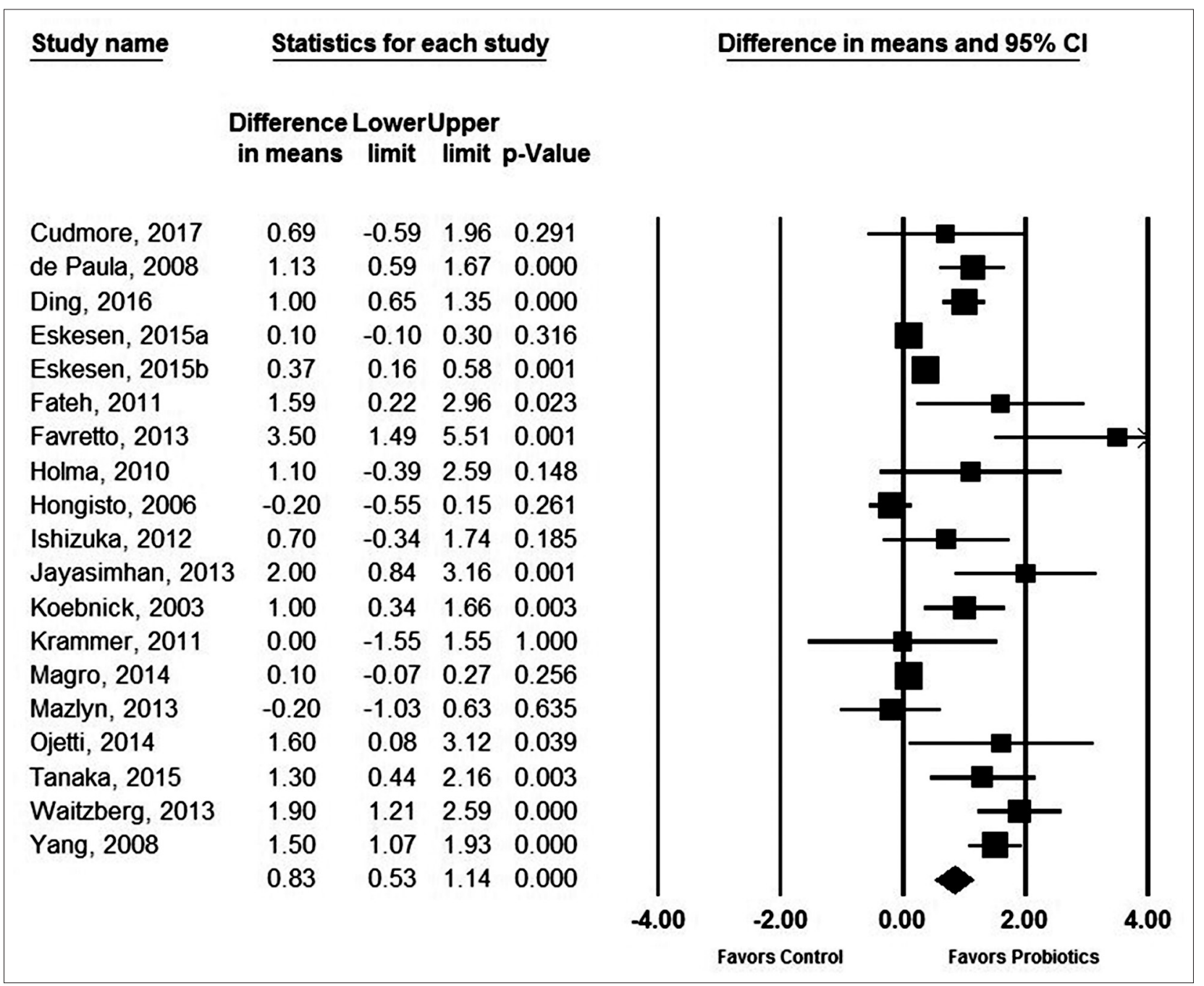

Figure 2 Forest plot of mean difference in weekly stool frequency with probiotics vs. controls. Pooled estimates derived from random effects model. Unit of measure is mean difference in bowel movements per week between probiotic and control. Heterogeneity: $I^{2}=85 \%, \mathrm{P}<0.001$ 


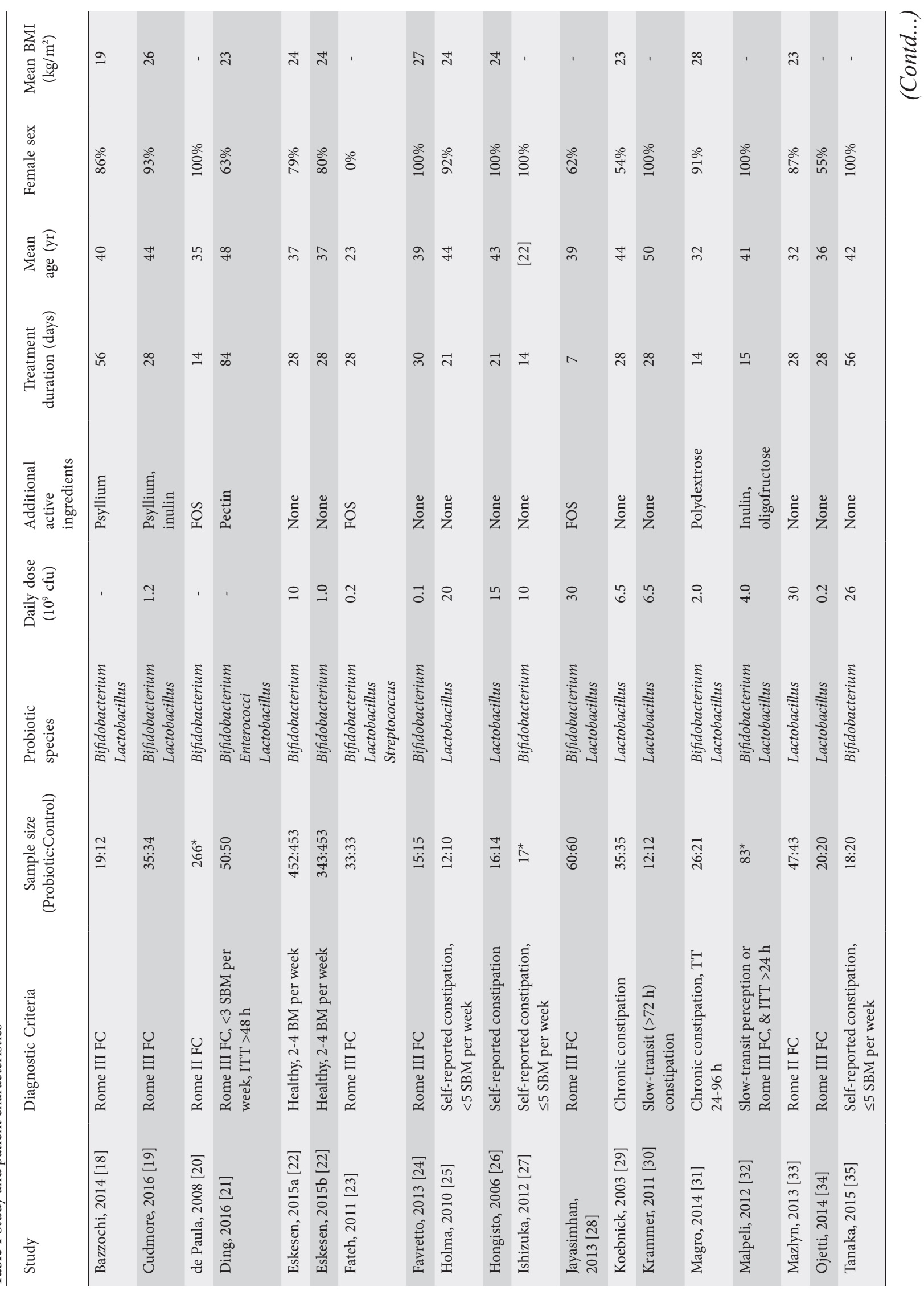




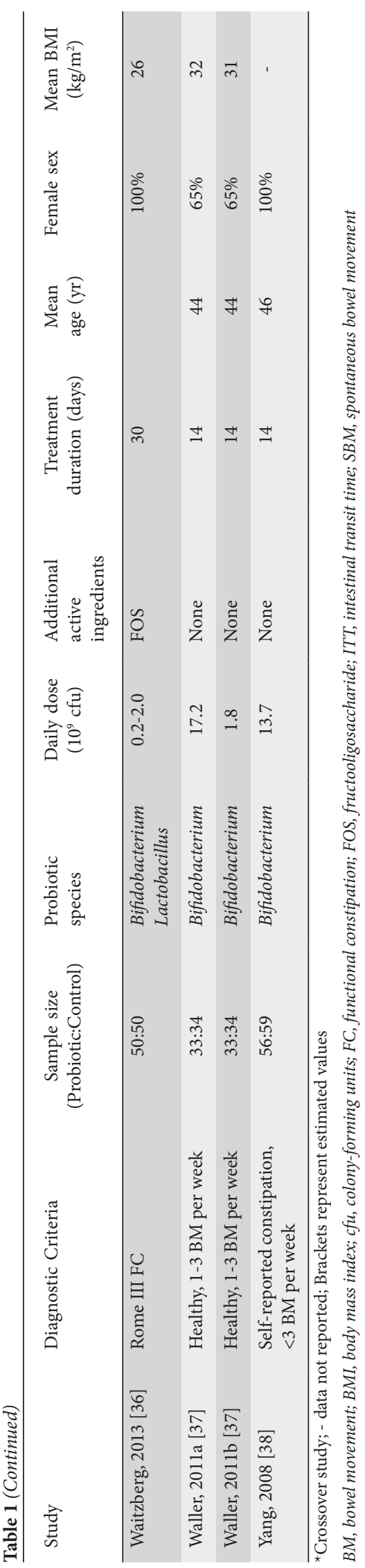

<0.01) (Fig. 3). After adjustment for publication bias, the mean difference in stool frequency was reduced from 0.83 0.30 . No single study significantly influenced the effect size of probiotics vs. controls on stool frequency. Removal of each study individually from the meta-analysis resulted in effect sizes ranging from $0.70-0.85$ (all $\mathrm{P}<0.001$ ). In meta-regression, the only variable that influenced the efficacy of probioticcontaining products was the use of the Rome functional constipation diagnostic criteria. Specifically, efficacy was greater in studies where functional constipation was diagnosed using Rome III $(\mathrm{P}<0.01)$, or Rome II or III criteria $(\mathrm{P}<0.05)$ compared to other diagnosis techniques (Table 3 ). The probiotic species, number of probiotic strains and daily probiotic dosage had no statistically significant influence on outcomes. The mean difference in weekly stool frequency was 1.14 (95\%CI $0.42-1.86, \mathrm{P}<0.01)$ for products containing Lactobacillus and Bifidobacterium, 0.93 (95\%CI 0.44-1.43, $\mathrm{P}<0.001)$ for products containing Bifidobacterium only, and 0.42 (95\%CI -0.22 to $1.07, \mathrm{P}=0.19$ ) for products containing Lactobacillus only. Meta-regression findings were not significantly affected by the influence of individual studies as all jackknifed residual values were within \pm 2.5 .

\section{Probiotic effects on ITT}

Probiotic-containing supplements resulted in a statistically significant improvement in ITT versus controls in 4 of 9 comparisons. When data were pooled in the meta-analysis, the SMD for ITT was 0.65 (95\%CI 0.33-0.97, P<0.001) (Fig. 4), analogous to a mean difference of $15(95 \% \mathrm{CI} 9-21) \mathrm{h}$. There was high heterogeneity among studies $\left(I^{2}=66 \%, \mathrm{P}<0.01\right)$, but no evidence of publication bias (Egger's P-value $=0.52$ ) (Fig. 5). No single study significantly influenced the effect size of probiotics vs. controls on ITT. The SMD after removal of each study individually from the meta-analysis ranged from 0.54-0.71 (all $\mathrm{P} \leq 0.001$ ). In meta-regression, a larger total sample size was the only variable that was associated with greater efficacy of probiotic-containing products with respect to ITT $(\mathrm{P}=0.03)$ (Table 3$)$. Neither probiotic species, number of probiotic strains, nor daily probiotic dosage statistically influenced outcomes. The SMD in ITT was 0.81 (95\%CI $0.20-1.41, \mathrm{P}<0.01)$ for products containing Lactobacillus and Bifidobacterium, 0.72 (95\%CI 0.37-1.07, $\mathrm{P}<0.001)$ for products containing Bifidobacterium only, and 0.27 (95\%CI -0.18 to $0.72, \mathrm{P}=0.24$ ) for products containing Lactobacillus only. Meta-regression findings were not significantly affected by the influence of individual studies as all jackknifed residual values were within \pm 2.4 .

\section{Discussion}

Significant heterogeneity in outcomes was detected among studies of probiotic preparations containing Lactobacillus or Bifidobacterium species in constipated adults. Therefore, the 


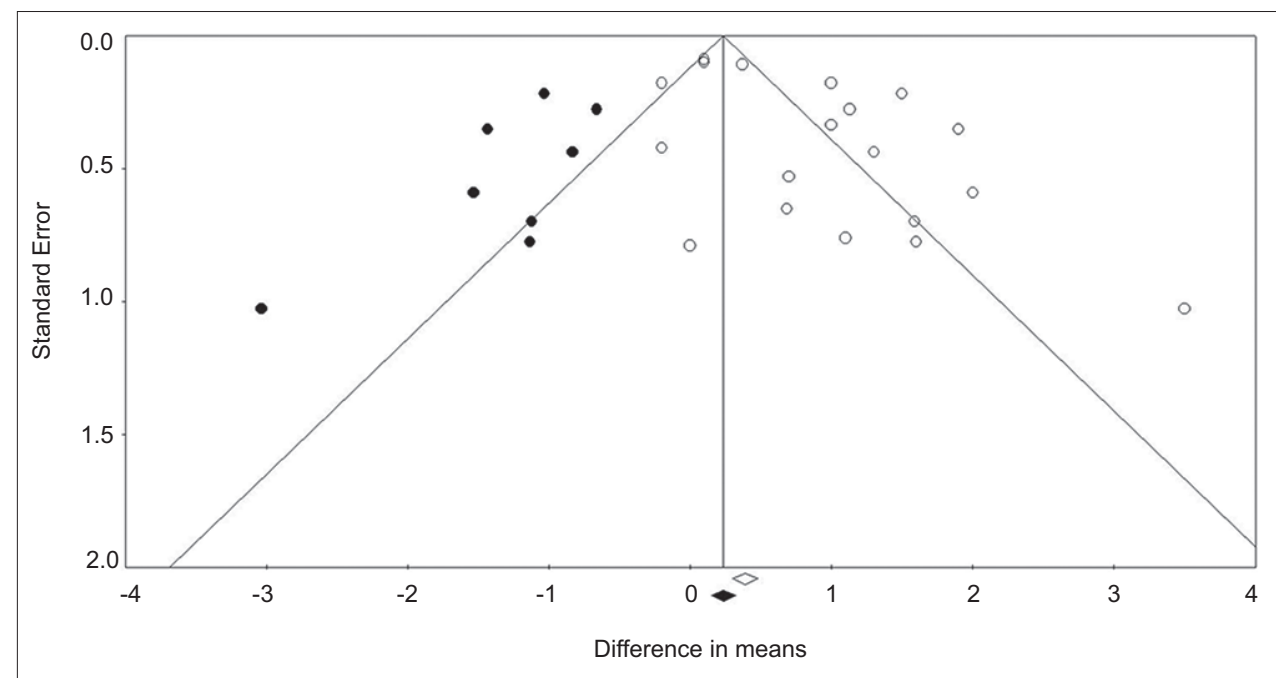

Figure 3 Funnel plot of mean difference in weekly stool frequency with probiotics vs. controls. Egger's P-value is $<0.01$, suggesting significant publication bias. Open circles represent observed values; shaded circles represent trim-and-fill adjusted values. Pooled estimate for observed values is 0.83 ( $95 \%$ confidence interval [CI] 0.53-1.14), represented by an open diamond. Pooled estimate for trim-and-fill adjusted values is 0.30 ( $95 \% \mathrm{CI}-0.01$ to 0.62 ), represented by a shaded diamond

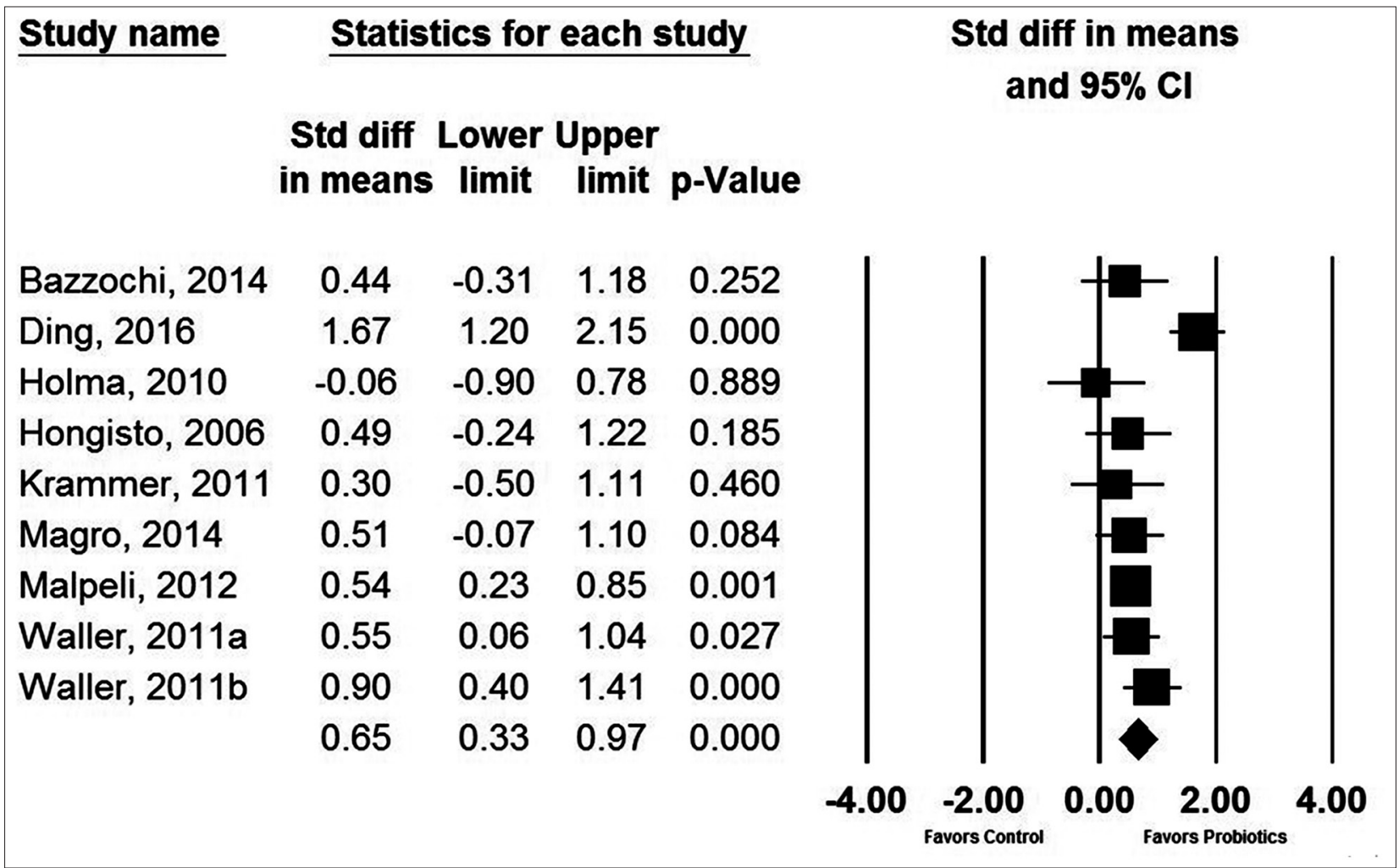

Figure 4 Forest plot of standardized mean difference in intestinal transit time with probiotics vs. controls. Pooled estimates derived from random effects model. Values of $0.2,0.5$, and 0.8 represent small, medium, and large effect sizes, respectively. Heterogeneity: $I^{2}=66 \%, \mathrm{P}<0.01$

detected efficacy of these products in increasing stool frequency and reducing ITT should be interpreted with caution. The main findings from this meta-analysis were that probiotics containing Lactobacillus or Bifidobacterium species increase stool frequency by approximately 0.8 bowel movements per week, although this is likely to be overestimated as a result of publication bias, and that probiotics decrease ITT by approximately $15 \mathrm{~h}$.

It is important to interpret the findings of this metaanalysis in light of previous reviews on the topic. In previous 
Table 2 Risk of bias assessment on the Jadad scale

\begin{tabular}{|c|c|c|c|c|}
\hline Study & $\begin{array}{l}\text { Randomization } \\
\text { Range: } 0 \text { to } 2\end{array}$ & $\begin{array}{c}\text { Double blinding } \\
\text { Range: } 0 \text { to } 2\end{array}$ & $\begin{array}{l}\text { Subject account } \\
\text { Range: } 0 \text { to } 1\end{array}$ & $\begin{array}{l}\text { Total score } \\
\text { Range: } 0 \text { to } 5\end{array}$ \\
\hline Bazzochi, 2014 [18] & 1 & 2 & 1 & 4 \\
\hline Cudmore, 2016 [19] & 1 & 2 & 1 & 4 \\
\hline de Paula, 2008 [20] & 1 & 0 & 1 & 2 \\
\hline Ding, 2016 [21] & 1 & 1 & 1 & 3 \\
\hline Eskesen, 2015a [22] & 2 & 2 & 1 & 5 \\
\hline Eskesen, 2015b [22] & 2 & 2 & 1 & 5 \\
\hline Fateh, 2011 [23] & 1 & 2 & 1 & 4 \\
\hline Favretto, 2013 [24] & 1 & 0 & 1 & 2 \\
\hline Holma, 2010 [25] & 1 & 0 & 1 & 2 \\
\hline Hongisto, 2006 [26] & 1 & 0 & 0 & 1 \\
\hline Ishizuka, 2012 [27] & 1 & 2 & 0 & 3 \\
\hline Jayasimhan, 2013 [28] & 1 & 2 & 1 & 4 \\
\hline Koebnick, 2003 [29] & 1 & 2 & 1 & 4 \\
\hline Krammer, 2011 [30] & 1 & 1 & 1 & 3 \\
\hline Magro, 2014 [31] & 2 & 2 & 1 & 5 \\
\hline Malpeli, 2012 [32] & 0 & 2 & 1 & 3 \\
\hline Mazlyn, 2013 [33] & 2 & 2 & 1 & 5 \\
\hline Ojetti, 2014 [34] & 2 & 2 & 1 & 5 \\
\hline Tanaka, 2015 [35] & 1 & 1 & 1 & 3 \\
\hline Waitzberg, 2013 [36] & 2 & 2 & 1 & 5 \\
\hline Waller, 2011a [37] & 2 & 2 & 1 & 5 \\
\hline Waller, 2011b [37] & 2 & 2 & 1 & 5 \\
\hline Yang, 2008 [38] & 1 & 1 & 1 & 3 \\
\hline
\end{tabular}

${ }^{\star}$ Higher scores represent better study quality

meta-analyses of randomized controlled trials, probiotics have been reported to increase stool frequency by 1.3-1.5 bowel movements per week relative to controls $[9,10]$. While our conclusions regarding probiotic efficacy are congruent with these previous reports, the estimated treatment effect in the current meta-analysis ( 0.8 bowel movements per week) is considerably lower. While each of the aforementioned metaanalyses included no more than 10 studies, the findings from our research are more comprehensive and contemporary with the inclusion of 20 comparisons from 19 studies. In agreement with the meta-analysis of Dimidi et al [37], we identified high heterogeneity among studies. Variability in stool frequency among studies was partially explained by the choice of diagnostic criteria in meta-regression. Subjects who received a diagnosis of functional constipation based on the Rome III criteria were considerably more responsive to probioticcontaining products than those diagnosed by self-report or physician opinion. While the importance of this finding as it relates to clinical practice is debatable, this may serve useful in clinical trial design, where investigators may attempt to control for potential sources of outcome variability, such as differences in the diagnostic criteria for constipation.
Regarding ITT, our findings were comparable to previous studies. We reported an SMD of 0.65 , corresponding to a decrease of $15 \mathrm{~h}$. These estimates are similar to the $12-\mathrm{h}$ reduction in ITT reported by Dimidi et al [37] and the SMD of 0.57 reported by Miller et al [39] in adults with constipation. While probiotic efficacy in relation to ITT was greater in larger studies, sample size was not associated with stool frequency. A Rome-based functional constipation diagnosis $(\mathrm{P}=0.06)$, a lower proportion of females $(\mathrm{P}=0.06)$, and a longer treatment duration $(\mathrm{P}=0.07)$ were associated with greater probiotic efficacy, but the associations did not reach statistical significance.

The major strengths of this research were the inclusion of only randomized controlled trials and the detailed evaluation of the impact of moderator variables, publication bias, and influential studies on the efficacy of probiotic-containing products. There were also several limitations inherent in the design of the included studies that warrant further elaboration. While study duration ranged from 1-12 weeks, only 2 studies followed subjects for more than 4 weeks. Consequently, assessment of the efficacy of probiotics in constipated adults over longer periods is warranted. Next, most studies included young to middle-aged adults with a predominance of women; therefore, future studies 
Table 3 Meta-regression of study- and subject-related factors on stool frequency and intestinal transit time

\begin{tabular}{|c|c|c|c|c|c|c|c|}
\hline \multirow[t]{2}{*}{ Variable } & \multirow[t]{2}{*}{ Unit of measure } & \multicolumn{3}{|c|}{ Stool frequency * } & \multicolumn{3}{|c|}{ Intestinal transit time $\dagger$} \\
\hline & & Intercept $\ddagger$ & Slope $(95 \% \mathrm{CI}) \ddagger$ & P-value & Intercept $\ddagger$ & Slope $(95 \%$ CI $) \ddagger$ & P-value \\
\hline \multirow[t]{2}{*}{ Diagnosis } & Rome III FC (1) vs. Other (0) & 0.543 & $1.021(0.297,1.744)$ & $<0.01$ & 0.521 & $0.707(-0.047,1.461)$ & 0.06 \\
\hline & Rome II/III FC (1) vs. Other (0) & 0.551 & $0.725(0.003,1.447)$ & $<0.05$ & 0.521 & $0.707(-0.047,1.461)$ & 0.06 \\
\hline \multirow{3}{*}{ Geography } & Americas (1) vs. Europe (0) & 0.461 & $0.743(-0.249,1.735)$ & 0.13 & 0.315 & $0.288(-0.266,0.843)$ & 0.25 \\
\hline & Asia (1) vs. Europe (0) & 0.461 & $0.632(-0.223,1.486)$ & 0.14 & $\$$ & $\$$ & $\$$ \\
\hline & Americas (1) vs. Asia (0) & 1.092 & $0.111(-0.902,1.125)$ & 0.82 & $\$$ & $\$$ & $\$$ \\
\hline \multirow{3}{*}{$\begin{array}{l}\text { Probiotic } \\
\text { species }\end{array}$} & Combination (1) vs. Lactobacillus (0) & 0.435 & $0.680(-0.336,1.696)$ & 0.17 & 0.258 & $0.557(-0.438,1.551)$ & 0.22 \\
\hline & Bifidobacterium (1) vs. Lactobacillus (0) & 0.435 & $0.531(-0.436,1.498)$ & 0.26 & 0.258 & $0.467(-0.678,1.613)$ & 0.36 \\
\hline & Combination (1) vs. Bifidobacterium (0) & 0.967 & $0.149(-0.781,1.080)$ & 0.74 & 0.725 & $0.089(-0.918,1.097)$ & 0.84 \\
\hline $\begin{array}{l}\text { Total } \\
\text { sample size }\end{array}$ & Per 10 subjects & 1.021 & $-0.008(-0.022,0.006)$ & 0.23 & -0.128 & $0.133(0.015,0.250)$ & 0.03 \\
\hline $\begin{array}{l}\text { Daily } \\
\text { probiotic } \\
\text { dosage }\end{array}$ & Per $1 \times 10^{10} \mathrm{cfu}$ & 0.524 & $-0.313(-0.948,0.321)$ & 0.27 & 0.668 & $-0.171(-0.572,0.231)$ & 0.32 \\
\hline $\begin{array}{l}\text { Probiotic } \\
\text { strains }\end{array}$ & Single strain (1) vs. multiple strain (0) & 1.117 & $-0.368(-1.214,0.479)$ & 0.37 & 0.814 & $-0.328(-1.118,0.463)$ & 0.36 \\
\hline $\begin{array}{l}\text { Treatment } \\
\text { duration }\end{array}$ & Per 1 day & -0.099 & $0.014(-0.006,0.033)$ & 0.39 & 0.299 & $0.013(-0.001,0.028)$ & 0.07 \\
\hline $\begin{array}{l}\text { Body mass } \\
\text { index }\end{array}$ & Per 1 kg/m² & -2.392 & $0.124(-0.256,0.504)$ & 0.48 & 0.751 & $-0.002(-0.137,0.132)$ & 0.97 \\
\hline $\begin{array}{l}\text { Female } \\
\text { gender } \\
\text { proportion }\end{array}$ & Per $10 \%$ & 1.366 & $-0.060(-0.238,0.118)$ & 0.49 & 2.249 & $-0.191(-0.396,0.015)$ & 0.06 \\
\hline Age & Per 10 years & -1.276 & $0.420(-0.999,1.840)$ & 0.50 & -0.634 & $0.300(-0.508,1.108)$ & 0.41 \\
\hline Jadad score & Per 1 point & 1.110 & $-0.065(-0.383,0.253)$ & 0.67 & 0.475 & $0.047(-0.269,0.363)$ & 0.73 \\
\hline $\begin{array}{l}\text { Stool } \\
\text { frequency }\end{array}$ & Per 1 bowel movement per week & 0.683 & $-0.017(-0.806,0.772)$ & 0.76 & $\$$ & $\$$ & $\$$ \\
\hline
\end{tabular}

${ }^{\star}$ Outcome is mean difference in bowel movements per week; †Outcome is standardized mean difference in intestinal transit time; $\ddagger$ Intercept represents estimated outcome value when covariate value $=0$. Slope represents the magnitude of change in estimated outcome value per unit increase in covariate value; \$Inadequate number of studies to perform meta-regression.

Note 1: Example calculation of outcome estimation using binary covariate where the outcome is the mean difference in stool frequency with probiotics vs. placebo and the covariate is Rome III diagnosis (yes or no). In subjects with diagnosis as Other, the mean difference is calculated as: [Intercept (0.543)] + [Slope $(1.021) *$ Indicator for Other $(0)]=0.543$ bowel movements per week. In subjects with diagnosis as Rome III, the mean difference is calculated as: [Intercept (0.543) $]^{*}$ [Slope (1.021)* Indicator for Rome III (1)] = 1.564 bowel movements per week

Note 2: Example calculation of outcome estimation using continuous covariate where the outcome is the mean difference in stool frequency with probiotics vs. placebo and the covariate is treatment duration in days. For a study of 20 days, the mean difference is calculated as: [Intercept $(-0.099)]+[$ Slope $(0.014)$ * Indicator $(20)]=0.181$ bowel movements per week

Cfu, colony forming units; FC, functional constipation; CI, confidence interval

of the topic in males and the elderly would fill an important gap in probiotic research. While meta-regression identified the method of diagnosing functional constipation as a factor that may influence probiotic efficacy, definitive conclusions cannot be drawn given the post hoc nature of the analysis. A range of probiotic-containing products were used in the studies, some also containing synbiotics and other dietary ingredients. While we attempted to characterize potential sources of heterogeneity in probiotic products by analyzing probiotic strain, number of strains, and probiotic dosage, we could not assess the influence of these other ingredients given the variation in usage among studies. Still, it is plausible that unmeasured variables related to the content of these products may influence probiotic efficacy. Finally, no eligible studies utilized the Rome IV diagnostic criteria, so the probiotic efficacy in patients diagnosed using these criteria is currently unknown.

In conclusion, a significant heterogeneity in outcomes was detected among studies of probiotic preparations containing Lactobacillus or Bifidobacterium species in constipated adults. Therefore, the detected efficacy of these products in increasing stool frequency and reducing ITT should be interpreted with caution. The main findings from this meta-analysis were that probiotics 


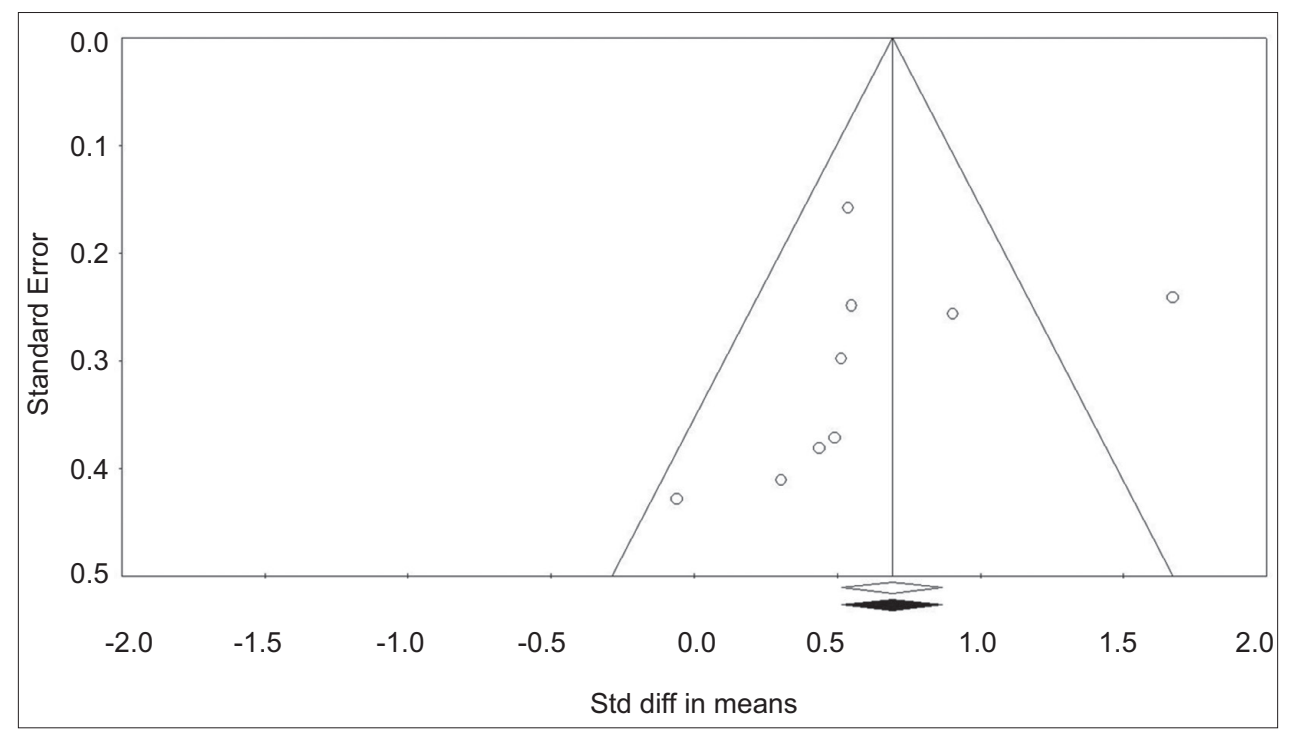

Figure 5 Funnel plot of standardized mean difference in intestinal transit time with probiotics vs. controls. Egger's P-value is 0.52 , suggesting no significant publication bias. Open circles represent observed values. No studies were imputed with the trim-and-fill method; therefore, the pooled estimate (standardized mean difference $=0.65,95 \%$ confidence interval $0.33-0.97$ ) is identical for observed values (represented by an open diamond) and imputed values (represented by a shaded diamond)

\section{Summary Box}

\section{What is already known:}

- Probiotics are commonly recommended for the alleviation of constipation symptoms

- The clinical effects of probiotics in adults with constipation are not well characterized

\section{What the new findings are:}

- Supplementation with products containing Lactobacillus or Bifidobacterium species increased stool frequency by approximately 0.8 bowel movements per week in constipated adults

- Supplementation with products containing Lactobacillus or Bifidobacterium species decreased intestinal transit time by approximately 15 hours in constipated adults

containing Lactobacillus or Bifidobacterium species increase stool frequency by approximately 0.8 bowel movements per week, although this is likely to be overestimated as a result of publication bias, and that probiotics decrease ITT by approximately $15 \mathrm{~h}$.

\section{Acknowledgment}

We thank David Fay, PhD, for assistance with the literature review.

\section{References}

1. Suares NC, Ford AC. Prevalence of, and risk factors for, chronic idiopathic constipation in the community: systematic review and meta-analysis. Am J Gastroenterol 2011;106:1582-1591.

2. Attaluri A, Jackson M, Valestin J, Rao SS. Methanogenic flora is associated with altered colonic transit but not stool characteristics in constipation without IBS. Am J Gastroenterol 2010;105:1407-1411.

3. Quigley EM. The enteric microbiota in the pathogenesis and management of constipation. Best Pract Res Clin Gastroenterol 2011;25:119-126.

4. Khalif IL, Quigley EM, Konovitch EA, Maximova ID. Alterations in the colonic flora and intestinal permeability and evidence of immune activation in chronic constipation. Dig Liver Dis 2005;37:838-849.

5. Barbara G, Stanghellini V, Brandi G, et al. Interactions between commensal bacteria and gut sensorimotor function in health and disease. Am J Gastroenterol 2005;100:2560-2568.

6. Malaguarnera G, Leggio F, Vacante M, et al. Probiotics in the gastrointestinal diseases of the elderly. $J$ Nutr Health Aging 2012;16:402-410.

7. Girardin M, Seidman EG. Indications for the use of probiotics in gastrointestinal diseases. Dig Dis 2011;29:574-587.

8. Jung HK. Rome III Criteria for functional gastrointestinal disorders: is there a need for a better definition? J Neurogastroenterol Motil 2011; 17:211-212.

9. Ford AC, Quigley EM, Lacy BE, et al. Efficacy of prebiotics, probiotics, and synbiotics in irritable bowel syndrome and chronic idiopathic constipation: systematic review and meta-analysis. Am J Gastroenterol 2014;109:1547-1561.

10. Dimidi E, Christodoulides S, Fragkos KC, Scott SM, Whelan K. The effect of probiotics on functional constipation in adults: a systematic review and meta-analysis of randomized controlled trials. Am J Clin Nutr 2014;100:1075-1084.

11. Liberati A, Altman DG, Tetzlaff J, et al. The PRISMA statement for reporting systematic reviews and meta-analyses of studies that evaluate health care interventions: explanation and elaboration. 
Ann Intern Med 2009; 151:W65-W94.

12. Jadad AR, Moore RA, Carroll D, et al. Assessing the quality of reports of randomized clinical trials: is blinding necessary? Control Clin Trials 1996;17:1-12.

13. Cohen J. Statistical power analysis for the behavioral sciences. Hillside, NJ: Lawrence Erlbaum Associates; 1987.

14. Higgins JPT, Green S. Cochrane Handbook for Systematic Reviews of Interventions. The Cochrane Collaboration; 2011. Available from: www.cochrane-handbook.org.

15. Higgins JP, Thompson SG, Deeks JJ, Altman DG. Measuring inconsistency in meta-analyses. BMJ 2003;327:557-560.

16. Egger M, Davey Smith G, Schneider M, Minder C. Bias in metaanalysis detected by a simple, graphical test. BMJ 1997;315:629-634.

17. Knapp G, Hartung J. Improved tests for a random effects metaregression with a single covariate. Stat Med 2003;22:2693-2710.

18. Bazzocchi G, Giovannini T, Giussani C, Brigidi P, Turroni S. Effect of a new synbiotic supplement on symptoms, stool consistency, intestinal transit time and gut microbiota in patients with severe functional constipation: a pilot randomized double-blind, controlled trial. Tech Coloproctol 2014;18:945-953.

19. Cudmore S, Doolan A, Lacey S, Shanahan F. A randomised, double-blind, placebo-controlled clinical study: the effects of a synbiotic, Lepicol, in adults with chronic, functional constipation. Int J Food Sci Nutr 2017;68:366-377.

20. De Paula JA, Carmuega E, Weill R. Effect of the ingestion of a symbiotic yogurt on the bowel habits of women with functional constipation. Acta Gastroenterol Latinoam 2008;38:16-25.

21. Ding C, Ge X, Zhang X, et al. Efficacy of synbiotics in patients with slow transit constipation: a prospective randomized trial. Nutrients 2016;8. pii: E605.

22. Eskesen D, Jespersen L, Michelsen B, Whorwell PJ, Müller-Lissner S, Morberg CM. Effect of the probiotic strain Bifidobacterium animalis subsp. lactis, $\mathrm{BB}-12^{\circ}$, on defecation frequency in healthy subjects with low defecation frequency and abdominal discomfort: a randomised, double-blind, placebo-controlled, parallel-group trial. Br J Nutr 2015;114:1638-1646.

23. Fateh R, Iravani S, Frootan M, Rasouli MR, Saadat S. Synbiotic preparation in men suffering from functional constipation: a randomised controlled trial. Swiss Med Wkly 2011;141:w13239.

24. Favretto DC, Pontin B, Moreira TR. Effect of the consumption of a cheese enriched with probiotic organisms (Bifidobacterium lactis bi-07) in improving symptoms of constipation. Arq Gastroenterol 2013;50:196-201.

25. Holma R, Hongisto SM, Saxelin M, Korpela R. Constipation is relieved more by rye bread than wheat bread or laxatives without increased adverse gastrointestinal effects. J Nutr 2010;140:534-541.

26. Hongisto SM, Paajanen L, Saxelin M, Korpela R. A combination of fibre-rich rye bread and yoghurt containing Lactobacillus GG improves bowel function in women with self-reported constipation.
Eur J Clin Nutr 2006;60:319-324.

27. Ishizuka A, Tomizuka K, Aoki R, et al. Effects of administration of Bifidobacterium animalis subsp. lactis GCL2505 on defecation frequency and bifidobacterial microbiota composition in humans. J Biosci Bioeng 2012;113:587-591.

28. Jayasimhan S, Yap NY, Roest Y, Rajandram R, Chin KF. Efficacy of microbial cell preparation in improving chronic constipation: a randomized, double-blind, placebo-controlled trial. Clin Nutr 2013;32:928-934.

29. Koebnick C, Wagner I, Leitzmann P, Stern U, Zunft HJ. Probiotic beverage containing Lactobacillus casei Shirota improves gastrointestinal symptoms in patients with chronic constipation. Can J Gastroenterol 2003;17:655-659.

30. Krammer HJ, von Seggern H, Schaumburg J, Neumer F. Effect of Lactobacillus casei Shirota on colonic transit time in patients with chronic constipation. Coloproctology 2011;33:109-113.

31. Magro DO, de Oliveira LM, Bernasconi I, et al. Effect of yogurt containing polydextrose, Lactobacillus acidophilus NCFM and Bifidobacterium lactis HN019: a randomized, double-blind, controlled study in chronic constipation. Nutr J 2014;13:75.

32. Malpeli A, González S, Vicentin D, Apás A, González HF. Randomised, double-blind and placebo-controlled study of the effect of a synbiotic dairy product on orocecal transit time in healthy adult women. Nutr Hosp 2012;27:1314-1319.

33. Mazlyn MM, Nagarajah LH, Fatimah A, Norimah AK, Goh KL. Effects of a probiotic fermented milk on functional constipation: a randomized, double-blind, placebo-controlled study. J Gastroenterol Hepatol 2013;28:1141-1147.

34. Ojetti V, Ianiro G, Tortora A, et al. The effect of Lactobacillus reuteri supplementation in adults with chronic functional constipation: a randomized, double-blind, placebo-controlled trial. J Gastrointestin Liver Dis 2014;23:387-391.

35. Tanaka Y, Takami K, Nishijima T, Aoki R, Mawatari T, Ikeda T. Short- and long-term dynamics in the intestinal microbiota following ingestion of Bifidobacterium animalis subsp. lactis GCL2505. Biosci Microbiota Food Health 2015;34:77-85.

36. Waitzberg DL, Logullo LC, Bittencourt AF, et al. Effect of synbiotic in constipated adult women - a randomized, double-blind, placebocontrolled study of clinical response. Clin Nutr 2013;32:27-33.

37. Waller PA, Gopal PK, Leyer GJ, et al. Dose-response effect of Bifidobacterium lactis HN019 on whole gut transit time and functional gastrointestinal symptoms in adults. Scand $J$ Gastroenterol 2011;46:1057-1064.

38. Yang YX, He M, Hu G, et al. Effect of a fermented milk containing Bifidobacterium lactis DN-173010 on Chinese constipated women. World J Gastroenterol 2008;14:6237-6243.

39. Miller LE, Zimmermann AK, Ouwehand AC. Contemporary metaanalysis of short-term probiotic consumption on gastrointestinal transit. World J Gastroenterol 2016;22:5122-5131. 